\title{
Theoretical characteristics of Though-Carrying Particle (TCP) and Thought Retaining Particle (TRP) are necessary for providing plausible possible answers to the typical specific questions raised by different learned reviewers
}

\author{
Dhananjay Pal \\ Email address: \\ dhananjay.pal123@gmail.com, paldhananjay46@yahoo.com
}

Pharmacy College, Bengal School of Technology, Sugandha-Delhi Road, Chuchura, Dist.-Hooghly, West Bengal, INDIA, PIN-712 102

\section{To cite this article:}

Dhananjay Pal. Theoretical Characteristics of Though-Carrying Particle (TCP) and Thought Retaining Particle (TRP) Are Necessary for Providing Plausible Possible Answers to the Typical Specific Questions Raised by Different Learned Reviewers. American Journal of Modern Physics. Vol. 2, No. 6, 2013, pp. 362-374. doi: 10.11648/j.ajmp.20130206.24

\begin{abstract}
A single field emerged at the origin of the universe, already containing within itself the blueprint of the physical universe. The primordial single field triggered the onset of the universe. Most physicists believe that a single super-force dominated the first instants of creation. Scientists have arrived at a simple but decisive conclusion that consciousness is very much a part of the universe, like other objects. Our consciousness model involving thought-carrying particle (TCP), thought retaining particle (TRP) and thought force (TF) signifies the existence of universal consciousness that exists along with the universe. This universal consciousness is a functional state of the universal mind (UM). This UM is evolved at the Big Bang from void. The UM is constituted by these TCP and TRP in the inherent presence of thought force (TF). Thought force (TF) is an expression of universal consciousness. The Thought force (TF) being the primordial quantum field functions as the original super-force. TF being the original super-force functions as the origin of all the fundamental fields. TCP is the carrier of thought force (TF) that, in turn, appears to be the origin of all the fields. The quantized energy $\left(\varepsilon_{T}\right)$ of TCP is responsible to cause the universal consciousness as well as the cosmic microwave background radiation temperature. The individual consciousness owes its origin to the universal consciousness created by the same $\varepsilon_{T}$. The same $\varepsilon_{T}$ is the energy responsible for generating thought force (TF). TF being an expression of the universal consciousness is applicable to any inanimate object as well as to any biological system (having thinking ability). The TF exerts its functions both in vitro and in vivo. Any matter as well as any individual mind is constituted by these TCP and TRP in the inherent presence of thought force (TF) in vitro and thought force (TF) in vivo and these TCP and TRP are originated from the same Void. We showed the existence of thought force in microcosm [TF (micro)] and thought force in macrocosm [TF (macro)]. This TF (micro) is theoretically found to be stronger than the strong nuclear force. TF (macro) is theoretically found to be weaker even than the gravitational force. Theoretical characteristics of TCP and TRP can provide possible answers to many typical questions.
\end{abstract}

Keywords: Void, Cosmic Microwave Background Radiation (CMBR), Universal Mind (UM), Thought Force (TF), Though-Carrying Particle (TCP), Thought Retaining Particle (TRP), Quantized Energy $\left(\boldsymbol{\varepsilon}_{T}\right)$ of TCP

\section{Introduction}

In Eastern philosophical traditions, consciousness is intrinsic to the universe, whereas in most Western views, consciousness is extrinsic, emerging from complex computation. How can these views be reconciled?

It is most relevant and important to indicate the names of various eminent physicists like Erwin Schrödinger, Eugene Wigner, Brian Josephson, John Wheeler, Roger Penrose, Henry P Stapp, Freeman J. Dyson, Paul Davies, David Bohm, Basil Hiley, Fritjof Capra, Fred Alan Wolf and Amit Goswami who have addressed the inclusion of consciousness in their work. Consciousness is to be taken into account. 
1.1. In contrast to the usual linear sequence of matter, body, life, brain, mind, consciousness, here the proposed cyclic sequence is first universal consciousness (a functional state of the universal mind), and then matter, body, life, brain, and regeneration of mind and consciousness. The evolution of life with mind and consciousness is possible purely due to the inherent existence of universal consciousness which exists along with the universe. The human nervous system is evolved to provide an appropriate material structure to individualize the universal consciousness, a characteristic of reality, pervading all manifestations.

Consciousness model of Pal et al [1-3] involving TCP, TRP and thought force $\left(\mathrm{T}_{\mathrm{F}}\right)$ signifies the existence of universal consciousness that exists along with the universe. Pal et al [3] showed that this universal consciousness is a functional state of Universal Mind (UM). Pal et al [3] explained that the UM is evolved at the Big Bang from the eternal Void. This Void, in turn, is the source of infinite energy. And this UM is a finer matter. The individual mind being a constituent of the UM is also a finer matter. The constituents of the UM and individual mind are the same. The ultimate constituents of matter and mind are the same as both mind and matter are aspects of one fundamental reality, which is called UM. The brain is the mediating link or interface between the individual mind and body.

Pal et al [3] explained that the constituents of the UM are the ultimate constituents of matter itself as everything in this universe is a manifestation of this UM. Pal et al [3] expressed that the UM is constituted by these TCP and TRP in the inherent presence of thought force $\left(\mathrm{T}_{\mathrm{F}}\right)$. Pal et al $[1,3]$ further explained that the ultimate constituents of matter and mind are these TCP and TRP in the inherent presence of thought force $\left(\mathrm{T}_{\mathrm{F}}\right)$ in vitro and thought force $\left(\mathrm{T}_{\mathrm{F}}\right)$ in vivo.

Physicists determined that underlying quantum fields give birth to elementary particles. Bhaumik [4] mentioned that Frank Wilczek pointed out, "In quantum field theory, the primary elements of reality are not individual particles, but underlying fields. Thus, for example, all electrons are but excitations of an underlying field, naturally called electric field". The same holds true for all the fundamental particles of which matter is made.

The existence of matter depends on the existence of force and vice versa. TCP cannot exist without TRP and vice versa. Many physicists believe that unifying all the forces, including gravity, into a single theory would require a phenomenon called super-symmetry. With supersymmetry, every fermion would have a boson twin, and vice-versa. The thought force $\left(\mathrm{T}_{\mathrm{F}}\right)$ is carried by the TCP in the presence of its super-symmetrical partner TRP. TCP that behaves like boson should accompany its supersymmetrical partner TRP that functions like fermion in the generalized simpler way. It is to be noted that these TCP and TRP function like wavicle: wave-particle duality.

\section{Thought Force}

Pal et al [1,3] and Pal [5] explained the existence of thought force $\left(\mathrm{T}_{\mathrm{F}}\right)$. Thought force $\left(\mathrm{T}_{\mathrm{F}}\right)$, an expression of the universal consciousness, is the primordial quantum field that, in turn, functions as the primary unified field. This $T_{F}$ being an expression of the universal consciousness is applicable to any inanimate object as well as to any biological system (having thinking ability). Thus the $T_{F}$ being an expression of the universal consciousness exerts its functions both in vitro and in vivo.

Physicists determined that underlying quantum fields give birth to elementary particles. Pal [5] expressed that the thought force $\left(\mathrm{T}_{\mathrm{F}}\right)$ is the primordial quantum field. Thought force $\left(T_{F}\right)$ being the primordial quantum field functions as the primary unified field. Thought force $\left(\mathrm{T}_{\mathrm{F}}\right)$ being the primordial quantum field gives birth to TRP that appears to be the origin of all the matter particles. TCP is the carrier of thought force $\left(\mathrm{T}_{\mathrm{F}}\right)$ that, in turn, appears to be the origin of all the fields. TCP thus appears to be the origin of all the field particles.

In a purpose to involve both the non-living and living systems of the world, Pal [5] has shown the existences of these TCP, TRP and thought force $\left(\mathrm{T}_{\mathrm{F}}\right)$ in vitro and thought force $\left(\mathrm{T}_{\mathrm{F}}\right)$ in vivo. Anyone can call this TCP by any other name, but as the highly developed living system will have to be evolved in the universe in the long run and as the thought of highly developed living system appears to be a kind of force to be called the thought force $\left(\mathrm{T}_{\mathrm{F}}\right)$ in vivo, we considered it is wise to call it as TCP. Further, as the universe exists along with the universal consciousness that, in turn, is created by the quantized energy $\left(\varepsilon_{T}\right)$ of TCP, we had to use the term TCP.

Pal (5) expressed that the non-living system of the world is governed by the thought force $\left(\mathrm{T}_{\mathrm{F}}\right)$ in vitro and this Thought force $\left(\mathrm{T}_{\mathrm{F}}\right)$ in vitro gives rise to $\mathrm{T}_{\mathrm{F}}$ (micro), SNF, EMF, WNF, GF and $\mathrm{T}_{\mathrm{F}}$ (macro) where $\mathrm{TF}$ (micro) = Thought force in microcosm, SNF $=$ Strong nuclear force, $\mathrm{EMF}=$ Electromagnetic force, $\mathrm{WNF}=$ Weak nuclear force, $\mathrm{GF}=$ Gravitational force and TF (macro) $=$ Thought force in macrocosm. It is to be noted here that $\mathrm{TF}$ (micro) is a stronger force than the SNF and TF (macro) is a weaker force even than the GF.

Pal (5) also expressed that the living system of the world is governed by the thought force $\left(\mathrm{T}_{\mathrm{F}}\right)$ in vivo and this Thought force $\left(\mathrm{T}_{\mathrm{F}}\right)$ in vivo is a type of force that represents the biological 'thought' which is the action of mind. This 'thought' being a type of force controls the 'thought processes' involving the firing of neurons through the quantum mechanical activities of these TCP and TRP in the presence of consciousness. Consciousness in living organisms is a process which involves the quantum mechanical activities of these TCP and TRP, the ultimate constituents of any matter as well as any mind in the inherent presence of thought force $\left(\mathrm{T}_{\mathrm{F}}\right)$ in vitro and the thought force $\left(\mathrm{T}_{\mathrm{F}}\right)$ in vivo as indicated by $\mathrm{Pal}$ et al [3]. This consciousness, in turn, is the quantized energy $\left(\varepsilon_{T}\right)$ of TCP. The thought force $\left(\mathrm{T}_{\mathrm{F}}\right)$ in vivo is demonstrated in numerous experiments in which thought has an effect on a physical process (often known as mind over matter). This biological 'thought' is a type of force that can cause movement. Controlling movement through thought alone is 
observed in several experiments conducted by many scientists as indicated by Pal (5). These experiments thus signify the existence of thought force $\left(\mathrm{T}_{\mathrm{F}}\right)$ in vivo.

Pal [5] explained the existence of $\mathrm{T}_{\mathrm{F}}$ (micro) (= Thought force in microcosm). This $T_{F}$ (micro) is the strongest interaction (a new class of 'extra strong' interaction). It is stronger than SNF (Strong Nuclear Force).

Pal [5] has also shown the existence of $\mathrm{T}_{\mathrm{F}}$ (macro) $(=$

$$
\mathcal{E}_{T}=4.384 \times 10^{-16} \mathrm{erg} \equiv 2.73 \times 10^{-4} \mathrm{eV} \equiv 2.73 \mathrm{~K} \cong C M B R \text { temperature } \equiv 2.725 \mathrm{~K} \text {. }
$$

This conversion of erg $\equiv \mathrm{eV} \equiv \mathrm{K}$ is given by Weisskopf [6] as follows:

$$
1 e r g \cong 0.6241807 \times 10^{12} \mathrm{eV} \text { and } 10^{-4} \mathrm{eV} \cong 1 \mathrm{~K}
$$

\section{What is Life?}

Life is a state of flux that is being maintained by a typical form of energy which is nothing but the consciousness itself. Pal et al [3] and Pal [7] showed that the quantized energy $\left(\varepsilon_{T}\right)$ of TCP is responsible to cause the universal consciousness as well as the cosmic microwave background radiation temperature. The individual consciousness owes its origin to the universal consciousness created by the same $\varepsilon_{T}$. Pal et al $[1,3]$ and Pal [7] explained that life may be defined as a state of functional manifestation of consciousness that, in turn, is the quantized energy ( $\mathcal{E}_{T}$ ) of TCP. This $\mathcal{E}_{T}$ represents universal consciousness.

Thus,

$$
\text { Life }=\mathrm{f}(\text { Consciousness })=\mathrm{f}\left(\mathcal{E}_{T}\right)
$$

where $\varepsilon_{T}=$ quantized energy of the TCP $=4.384 \times 10^{-16} \mathrm{erg}$.

$$
\varepsilon_{T}=h v_{T}=h c / \lambda_{T}=4.384 \times 10^{-16} \mathrm{erg}
$$

where $\varepsilon_{T}=$ quantized energy of the TCP $=4.384 \times 10^{-16} \mathrm{erg}$,

$$
\boldsymbol{v}_{T}=\text { frequency of the TCP }=\varepsilon_{T} / h=66.12 \times 10^{9} \mathrm{~Hz} .=66.12 \mathrm{GHz},
$$

$\mathrm{h}=$ Planck's quantum constant $=6.63 \times 10^{-27} \mathrm{erg} . \mathrm{sec}$,

$\mathrm{c}=$ free-space velocity of light $=3 \times 10^{10} \mathrm{~cm} / \mathrm{sec}$,

$\lambda_{T}=$ wave-length of the $\mathrm{TCP}=0.4537 \mathrm{~cm}$.

\subsection{What is Consciousness?}

Psychologists, neuroscientists, philosophers, and other professionals continue to engage in an ongoing debate as to what consciousness means. In reality, we may never know. Is it a product of the biological and classical physical interactions of the human brain; or is it something more fundamental, perhaps electromagnetic, or the result of quantum physics principles that we don't yet fully understand? Could it be something even more profound than that -- something beyond the scope of science and physics, any kind of physics, for us to understand?

According to the ancient Vedanta, consciousness is not an emergent property of matter that comes into existence only through the functioning of the human nervous system. Instead, consciousness is a characteristic of reality, pervading all manifestations. This unbounded field of nature's universal consciousness is not limited to an individual consciousness. From this viewpoint, the role of the human nervous system is to provide an appropriate material structure to individualize the universal consciousness. It appears that inanimate matter itself cannot generate consciousness without the inherent existence of universal consciousness.

Pal et al [2] explained, "Consciousness is the realization of existence, and there are as many states of consciousness as there are states of existence. Every living being has a consciousness of its own depending on complexity of the brain and the activity of the viable numbers of TCP in the presence of TRP; and the state of its consciousness changes every moment of time. Consciousness is the perception of the relation it bears to things and as this relation changes, consciousness changes its character. Consciousness itself does not change; it only moves up and down on the 'scale of the realization' of existence through the 'sub-conscious', 'conscious' and 'super-conscious' states". There is another term called unconscious state. The most usual unconscious state is sleep. A deeper form of unconsciousness is called a coma.

\subsection{Consciousness May be Defined as the 'Self- Organized' Capability of any Living being to Activate TCP and TRP}

Pal et al [3] expressed that consciousness may be defined as the 'self-organized' capability of any living being to activate TCP and TRP, the ultimate constituents of mind and matter and to exert its functions. What can generate, maintain and activate TCP and TRP is called animate having 'active consciousness' and what cannot is called inanimate, i.e., devoid of active consciousness. As anything inanimate does neither have the power to activate the TCP and TRP nor have the capability to catalyze the activity of TCP and TRP, so it cannot generate consciousness. On the contrary, anything animate has the 'self-organized' power 
to generate, activate and catalyze the activity of TCP and TRP in order to generate and maintain consciousness along with the vital living force. Prigogine et al [8] explained "Self-Organization in Non-Equilibrium Systems".

Consciousness in living organisms is a process which involves the quantum mechanical activities of these TCP and TRP, the ultimate constituents of any matter as well as any mind in the inherent presence of thought force $\left(\mathrm{T}_{\mathrm{F}}\right)$ in vitro and the thought force $\left(\mathrm{T}_{\mathrm{F}}\right)$ in vivo as indicated by $\mathrm{Pal}$ et al $[1,3]$. And these TCP and TRP govern the activities of neurons (not the other way round). Neurons are simply the equipments used to generate consciousness and awareness. The consciousness itself is functioning as an inter-linking agent between the animate and inanimate through the quantum mechanical activities of these TCP and TRP indicated by [3].

\subsection{Possible Relation of Consciousness with Mind}

Modern scientists have not even been able to arrive at a consensus on what should be a definition of the totality of consciousness. The brain is intricately linked to the process of consciousness and consciousness is thought to be a phenomenon of the mind.

It appears that

$$
\text { Consciousness }=\mathrm{f}(\operatorname{mind})
$$

Consciousness is the functional state of mind. Presence of consciousness signifies the presence of mind and vice versa.

It is apparent that

$$
\text { Animate } \neq \text { Inanimate }
$$

Three critical factors which distinguish life from nonliving are consciousness, metabolism and reproduction. (4):

In a gross presentation, we can express from the equation

$$
\text { Animate }- \text { Consciousness }=\text { Inanimate }
$$

Scientists would have to define and characterize consciousness properly. We should have the proper knowledge about the exact characteristics of consciousness in order to address many present day scientific enigmas.

\subsection{Existence of Universal Consciousness}

Pal et al [3] and Pal [7] showed the existence of universal consciousness and explained that the quantized energy $\left(\varepsilon_{T}\right)$ of TCP is responsible to cause the universal consciousness as well as the cosmic microwave background radiation temperature. The individual consciousness owes its origin to the universal consciousness created by the same $\varepsilon_{T}$. Many physicists agree with the idea that consciousness is non-local, fundamental in the universe and consciousness is very much a part of the universe, like other objects. It is to be noted that $\varepsilon_{T}$, the quantized energy of TCP represents universal consciousness.
Nelson [9], Director of Global Consciousness Project, has observed through the experimentation that coherent consciousness creates order in the world; and subtle interactions link us with each other and the Earth. When human consciousness becomes coherent and synchronized, the behavior of random systems may change. Quantum event based random number generators (RNGs) produce completely unpredictable sequences of zeroes and ones. But when a great event synchronizes the feelings of millions of people, our network of RNGs becomes subtly structured. The probability is less than one in a billion that the effect is due to chance. The evidence suggests an emerging noosphere, or the unifying field of consciousness described by sages in all cultures. This evidence signifies and proves the existence of universal consciousness.

The existence of universal consciousness is explained by Radin [10] through experimentation as it is expressed in his book The Conscious Universe: The Scientific Truth of Psychic Phenomena. Further, Consciousness, not matter, is the ground of all existence, declares University of Oregon physicist Goswami through his published (1993) book, "The Self-Aware Universe: How Consciousness Creates the Material World".

As per Penrose [11], consciousness is a part of the universe. Van De Bogart [12] explained, "Since consciousness is a part of the universe it then follows that all consciousness, and the universe, are of the same matrix of energy fields".

\section{Theoretical Characteristics of TCP in the Presence of TRP (through Calculation): They are Necessary for Providing Answers to Many Typical Questions}

The existence of matter depends on the existence of force and vice versa. TCP cannot exist without TRP and vice versa. Many physicists believe that unifying all the forces, including gravity, into a single theory would require a phenomenon called super-symmetry. With supersymmetry, every fermion would have a boson twin, and vice-versa. The thought force $\left(\mathrm{T}_{\mathrm{F}}\right)$ is carried by the TCP in the presence of its super-symmetrical partner TRP. TCP that behaves like boson should accompany its supersymmetrical partner TRP that functions like fermion in the generalized simpler way. It is to be noted that these TCP and TRP function like wavicle: wave-particle duality.

The essential conversion chart along with the other universal constants is as follows

$$
\begin{aligned}
& 1 \mathrm{eV} \cong 1.6021 \times 10^{-12} \mathrm{erg} \cong 1.6021 \times 10^{-19} \mathrm{~J}=1 / 8066 \lambda= \\
& 23,060 \mathrm{kcal} / \mathrm{mole} \\
& 1 \mathrm{erg} \cong 0.6241807 \times 10^{12} \mathrm{eV} \cong 0.6241807 \times 10^{6} \mathrm{MeV} \cong \\
& 0.6241807 \times 10^{3} \mathrm{GeV}
\end{aligned}
$$$$
1 \mathrm{cal} \cong 4.186 \times 10^{7} \mathrm{erg} \cong 4.186 \mathrm{~J} \cong 2.61282 \times 10^{13} \mathrm{MeV}
$$ 
$1 \mathrm{erg} \cong 2.388915 \times 10^{-8} \mathrm{cal}$

$\mathrm{h}=$ Planck's quantum constant $=6.63 \times 10^{-27} \mathrm{erg} . \mathrm{sec}$

$\mathrm{c}=$ free-space velocity of light $=3 \times 10^{10} \mathrm{~cm} / \mathrm{sec}$

$\mathrm{G}=$ Gravitational constant $=6.67 \times 10^{-8}$ dyne. $\mathrm{cm}^{2} . \mathrm{g}^{-2}$

Basis of calculation: Radiant mass of the Universe through Stephan-Boltzmann formula indicated by Gamow $[13]=\mathrm{V}_{\mathrm{pr}} \rho_{\mathrm{r}}=1.16025 \times 10^{53} \mathrm{~g}$

Quantized energy $\left(\varepsilon_{T}\right)$ of a TCP in the presence of TRP $=$ $4.95 \times 10^{-16} \mathrm{erg} \cong 3.0897 \times 10^{-4} \mathrm{eV} \cong 3.0897 \times 10^{-10} \mathrm{MeV}$ $\cong 3.0897 \times 10^{-13} \mathrm{GeV} \cong 11.82513 \times 10^{-24} \mathrm{cal}$

$\mathcal{E}_{T}=\mathrm{hc} / \lambda_{\mathrm{T}}=\left(\mathrm{h}^{3} \mathrm{c}^{5} \mathrm{~m} / \mathrm{V}_{\mathrm{pr}}\right)^{1 / 4}=4.95 \times 10^{-16} \mathrm{erg}$

$\lambda_{\mathrm{T}}=\mathrm{hc} / \mathcal{E}_{T}=\left(\mathrm{hV}_{\mathrm{pr}} / \mathrm{mc}\right)^{1 / 4}=0.4018 \mathrm{~cm}$

$\mathrm{m}_{\mathrm{T}}=\varepsilon_{T} / \mathrm{c}^{2}=\left(\mathrm{h}^{3} \mathrm{~m} / \mathrm{c}^{3} \mathrm{~V}_{\mathrm{pr}}\right)^{1 / 4}=5.5 \times 10^{-37} \mathrm{~g}$

$\tau_{\mathrm{T}}=\lambda_{\mathrm{T}} / \mathrm{c}=\left(\mathrm{hV}_{\mathrm{pr}} / \mathrm{c}^{5} \mathrm{~m}\right)^{1 / 4}=1.34 \times 10^{-11} \mathrm{sec}$

where, $\mathrm{m}=$ radiant mass of the universe $=\mathrm{V}_{\mathrm{pr}} \cdot \rho_{\mathrm{r}}=$ $1.16025 \times 10^{53} \mathrm{~g}$

$\rho_{\mathrm{r}}=$ density of the radiant energy according to Stephan-

Boltzmann formula $=8.5 \times 10^{-36} \mathrm{~T}^{4} \mathrm{~g} / \mathrm{cm}^{3}$,

$\mathrm{h}=$ Planck's quantum constant $=6.63 \times 10^{-27}$ erg .sec,

$\mathrm{V}_{\mathrm{pr}}=$ present volume of the universe $=(4 / 3) \pi\left(\mathrm{c} / \mathrm{H}_{\mathrm{o}}\right)^{3}=$

$1.365 \times 10^{88} \mathrm{~cm}^{3}$

$\mathrm{H}_{\mathrm{o}}=$ present value of Hubble's parameter $=\mathrm{c} / \mathrm{L}$

$=\mathrm{c} / \mathrm{R}=2.023988 \times 10^{-19} \mathrm{sec}^{-1}$

$\mathrm{c}=$ free-space velocity of light $=3 \times 10^{10} \mathrm{~cm} / \mathrm{sec}$,

$\mathrm{c} / \mathrm{H}_{\mathrm{o}}=\mathrm{L}$ (Hubble length) $=$ radius of the universe $=\mathrm{R}$ $=1.482 \times 10^{29} \mathrm{~cm}$,

The radius of a TCP in the presence of TRP within a nucleon $\left(\mathrm{r}_{\mathrm{Tn}}\right)=8.28 \times 10^{-18} \mathrm{~cm}$.

$\rho_{\text {Tn }}=\rho_{\mathrm{n}}=\mathrm{m}_{\mathrm{n}} / \mathrm{V}_{\mathrm{n}}=\mathrm{m}_{\mathrm{n}} /(4 / 3) \pi\left(\mathrm{r}_{\mathrm{n}}\right)^{3}=0.231179 \times 10^{15} \mathrm{~g} / \mathrm{cm}^{3}$ where $\rho_{\mathrm{Tn}}=$ density, i.e. , concentration of the TRP in the presence of TCP within the structure of a nucleon,

$\rho_{\mathrm{n}}=$ density of a nucleon,

$\mathrm{m}_{\mathrm{n}}=$ rest mass of a nucleon $\approx 1.674 \times 10^{-24} \mathrm{~g}$,

$\mathrm{V}_{\mathrm{n}}=$ volume of a nucleon $=7.24 \times 10^{-39} \mathrm{~cm}^{3}$,

$\mathrm{r}_{\mathrm{n}}=$ radius of a nucleon $=1.2 \times 10^{-13} \mathrm{~cm}$,

When $\rho_{\mathrm{Tn}}=\rho_{\mathrm{n}}=0.231179 \times 10^{15} \mathrm{~g} / \mathrm{cm}^{3}$,

Volume of a TCP in the presence of TRP $\left(\mathrm{V}_{\mathrm{T}}\right)=\mathrm{m}_{\mathrm{T}} / \rho_{\mathrm{Tn}}=$
$\mathrm{m}_{\mathrm{T}} / \mathrm{\rho}_{\mathrm{n}}=(4 / 3) \pi\left(\mathrm{r}_{\mathrm{Tn}}\right)^{3}=2.38 \times 10^{-51} \mathrm{~cm}^{3}$

where $\mathrm{m}_{\mathrm{T}}=$ quantized mass of a TCP in the presence of

$\mathrm{TRP} \approx 5.5 \times 10^{-37} \mathrm{~g}$,

$\mathrm{r}_{\mathrm{Tn}}=$ radius of a TCP in the presence of TRP within a nucleon .

Thus, the radius of a TCP in the presence of TRP within a nucleon $\left(\mathrm{r}_{\mathrm{Tn}}\right)=8.28 \times 10^{-18} \mathrm{~cm}$.

Again, $\rho_{\mathrm{Te}}=\rho_{\mathrm{e}}=\mathrm{m}_{\mathrm{e}} / \mathrm{V}_{\mathrm{e}}=\mathrm{m}_{\mathrm{e}} /(4 / 3) \pi\left(\mathrm{r}_{\mathrm{e}}\right)^{3}$

$=0.1391345 \times 10^{11} \mathrm{~g} / \mathrm{cm}^{3}$

where $\rho_{\mathrm{Te}}=$ density, i.e., concentration of the TRP in the presence of TCP within the structure of an electron,

$\rho_{\mathrm{e}}=$ density of an electron,

$\mathrm{m}_{\mathrm{e}}=$ rest mass of an electron $=9.11 \times 10^{-28} \mathrm{~g}$,

$\mathrm{V}_{\mathrm{e}}=$ volume of an electron $=65.476 \times 10^{-39} \mathrm{~cm}^{3}$,

$r_{e}=$ radius of an electron $=2.5 \times 10^{-13} \mathrm{~cm}$,

Similarly, when $\rho_{\mathrm{Te}}=\rho_{\mathrm{e}}=0.1391345 \times 10^{11} \mathrm{~g} / \mathrm{cm}^{3}$,

$\mathrm{V}_{\mathrm{T}}=\mathrm{m}_{\mathrm{T}} / \rho_{\mathrm{Te}}=\mathrm{m}_{\mathrm{T}} / \rho_{\mathrm{e}}=(4 / 3) \pi\left(\mathrm{r}_{\mathrm{Te}}\right)^{3}=3.953 \times 10^{-47} \mathrm{~cm}^{3}$

Thus, the radius of a TCP in the presence of TRP within an electron $\left(\mathrm{r}_{\mathrm{Te}}\right)=2.113 \times 10^{-16} \mathrm{~cm}$.

$v($ frequency of a TCP in the presence of TRP $)=7.466 \mathrm{x}$ $10^{10} \mathrm{cps}$

$\lambda_{\mathrm{c}}($ Compton length $)=\mathrm{h} / \mathrm{m}_{\mathrm{T}} \mathrm{c}=0.4018 \mathrm{~cm}=\lambda_{\mathrm{T}}$

Gravitational length of a TCP in the presence of TRP $=$ $\mathrm{Gm}_{\mathrm{T}} / \mathrm{c}^{2}=4.0761 \times 10^{-65} \mathrm{~cm}$

$\mathrm{Sp}$. heat of a TCP in the presence of TRP $=2.15 \times 10^{13}$ $\mathrm{cal} / \mathrm{g}^{0} \mathrm{c}$

$1 \mathrm{gm}$ of TCP in the presence of TRP $\cong 1.81818 \times 10^{36} \mathrm{TCP}$ in the presence of $T R P \cong 1$ gm of any element

For the present universe of $10^{57} \mathrm{~g}$, the number of TCP in the presence of $\mathrm{TRP} \cong 1.81818 \times 10^{93}$

The Details of $T_{F}$ (Micro) are as Follows:

$$
\begin{aligned}
& T_{F}(\text { micro }) \cong 10^{5} S N F \cong 10^{44} \mathrm{GF} \cong 52.94 \text { dyne } \\
& 33.04 \times 10^{12} \mathrm{eV} \cong 33.04 \times 10^{3} \mathrm{GeV} \cong
\end{aligned}
$$

Strongest interaction (at $D_{i}=10^{-18} \mathrm{~cm}$, the theoretically calculated radius of a TCP or TRP within a nucleon) where $\mathrm{T}_{\mathrm{F}}$ (micro) $=$ Thought force in microcosm.

The details of TF (Macro) are as Follows:

$$
\begin{aligned}
& T_{F}(\text { macro }) \cong 10^{-3} G F \cong 10^{-42} S N F \\
& \cong 2.958 \times 10^{-45} d y n e \cong 1.846 \times 10^{-33} \mathrm{eV} \\
& \cong 1.846 \times 10^{-42} \mathrm{GeV} \cong
\end{aligned}
$$


Long-range weakest interaction [at $D_{i}$ (Interacting distance) $=$ radius of the universe $\approx 1.482 \times 10^{29} \mathrm{~cm}$ ] where $\mathrm{T}_{\mathrm{F}}(\mathrm{macro})=$ Thought force in macrocosm.

Table-I: Theoretical characteristics of TCP in the presence of TRP

$$
\begin{aligned}
& \varepsilon_{T}=\text { quantized energy of the TCP }=\left(\frac{h c}{\lambda_{T}}\right)=\left(\frac{h^{3} c^{5} m}{V_{p r}}\right)^{1 / 4}=4.95 \times 10^{-16} \mathrm{erg}, \\
& \mathrm{m}_{\mathrm{T}}=\text { quantized mass of the TCP }=\varepsilon_{T} / c^{2}=\left(\frac{h^{3} m}{c^{3} V_{p r}}\right)^{1 / 4}=5.5 \times 10^{-37} \mathrm{~g},
\end{aligned}
$$

Thus, $\mathcal{E}_{T}$, the quantized energy of the TCP radiated from the radiant mass of the universe is $\mathcal{E}_{T}=4.95 \times 10^{-16} \mathrm{erg}$ $\equiv 3.08 \times 10^{-4} \mathrm{eV} \equiv 3.08 \mathrm{~K}$

This conversion of erg $\equiv \mathrm{eV} \equiv \mathrm{K}$ is given by Weisskopf [6] as follows:

$$
1 e r g \cong 0.6241807 \times 10^{12} \mathrm{eV}^{\text {and }} 10^{-4} \mathrm{eV} \cong 1 \mathrm{~K}
$$

\section{Development of second equation}

$$
\varepsilon_{T}=\left(\frac{1}{4 \pi}\right)\left(\frac{h^{3} c^{8}}{G H_{0} V_{p r}}\right)^{1 / 4}=4.7142 \times 10-16 \mathrm{erg}
$$

where $\mathrm{G}=$ Gravitational constant $=6.67 \times 10^{-8}$ dyne. $\mathrm{cm}^{2} . \mathrm{g}^{-2}$

Here $\mathcal{E}_{T}=4.7142 \times 10^{-16} \mathrm{erg} \equiv 2.9 \times 10^{-4} \mathrm{eV} \equiv 2.9^{\circ} \mathrm{K} \cong \mathrm{CMBR}$ temperature $\equiv 2.725^{0} \mathrm{~K}$.

$$
\mathrm{m}_{\mathrm{T}}=\text { quantized mass of the TCP }=\varepsilon_{T} / c^{2}=\left(\frac{h^{3} m}{c^{3} V_{p r}}\right)^{1 / 4}=5.236 \times 10^{-37} \mathrm{~g},
$$

\section{Development of third equation}

$$
\varepsilon_{T}=\left(\frac{1}{(4+3 / 10) \pi}\right)\left(\frac{h^{3} c^{8}}{G H_{0} V_{p r}}\right)^{1 / 4}=4.384 \times 10-16 \mathrm{erg}
$$

Thus,

$$
\begin{aligned}
& \mathcal{E}_{T}=4.384 \times 10^{-16} \mathrm{erg} \equiv 2.73 \times 10^{-4} \mathrm{eV} \equiv 2.73 \mathrm{~K} \cong \mathrm{CMBR} \text { temperature } \equiv 2.725 \mathrm{~K} \\
& \mathrm{~m}_{\mathrm{T}}=\text { quantized mass of the } \mathrm{TCP}=\varepsilon_{T} / c^{2}=\left(\frac{h^{3} m}{c^{3} V_{p r}}\right)^{1 / 4}=4.871 \times 10^{-37} \mathrm{~g}
\end{aligned}
$$

Thus from the three developed equations, the quantized energy $\left(\mathcal{E}_{T}\right)$ of TCP is found to have a value ranging from $4.384 \times 10^{-16} \mathrm{erg}$ to $4.95 \times 10^{-16} \mathrm{erg}$.

Although it is assumed that these TCP and TRP exist in the universe to behave, in general, like photons or biophotons as indicated by $\mathrm{Pal}$ et al [3] for causing the universal consciousness as well as the Cosmic microwave background radiation (CMBR) temperature, yet they may be mathematically allotted a mass $\left(\mathrm{m}_{\mathrm{T}}\right)$ equivalent to $4.871 \times 10^{-37} \mathrm{~g}$ to $5.5 \times 10^{-37} \mathrm{~g}$.

4.2. Pal et al [3] developed three different equations expressing the quantized energy ( $\varepsilon_{T}$ ) of TCP.

The value of $\varepsilon_{T}$ in one of the three equations is shown below: 


$$
\varepsilon_{T}=4.384 \times 10^{-16} \mathrm{erg} \equiv 2.73 \times 10^{-4} \mathrm{eV} \equiv 2.73 \mathrm{~K} \cong C M B R \text { temperature } \equiv 2.725 \mathrm{~K} .
$$

This conversion of erg $\equiv \mathrm{eV} \equiv \mathrm{K}$ is given by Weisskopf [6] as follows:

$$
1 \mathrm{erg} \cong 0.6241807 \times 10^{12} \mathrm{eV} \text { and } 10^{-4} \mathrm{eV} \cong 1 \mathrm{~K}
$$

4.3. Pal et al [3] expressed that the CMBR temperature is due to $\varepsilon_{T}$, the quantized energy of TCP where

$$
\mathcal{E}_{T}=4.384 \times 10^{-16} \mathrm{erg} \equiv 2.73 \times 10^{-4} \mathrm{eV} \equiv 2.73 \mathrm{~K} \cong C M B R \text { temperature } \equiv 2.725 \mathrm{~K} .
$$

As per Pal et al [3], it is interesting to note that all of the three different equations ultimately give rise to the same result that is equivalent to the CMBR temperature. A sort of relationship is thus observed between the $\varepsilon_{T}$ and the CMBR temperature. This coincidence is thus signifying a probable role of TCP on the maintenance of CMBR temperature. Further, this coincidence is also signifying the existence of these TCP in the presence of TRP. TCP cannot exist without TRP and vice versa. The presence of TCP, TRP and Thought force $\left(\mathrm{T}_{\mathrm{F}}\right)$ in the universe is thus indicated and expressed mathematically. At present we are unable to explain when and how these TCP and TRP decoupled from the primordial cosmic soup.

Pal et al $[1,3]$ showed that the quantized energy $\left(\varepsilon_{T}\right)$ of TCP is responsible to cause the universal consciousness as well as the cosmic microwave background radiation temperature. The individual consciousness owes its origin to the universal consciousness created by the same $\varepsilon_{T}$. Ultimately this $\mathcal{E}_{T}$ represents universal consciousness. The existence of CMBR temperature indicates the existence of the TCP in the presence of TRP. The existence of TCP ensures the existence of the thought force $\left(\mathrm{T}_{\mathrm{F}}\right)$. Further, this $\mathrm{T}_{\mathrm{F}}$ being an expression of the quantized energy $\left(\mathcal{E}_{T}\right)$ of TCP exerts its functions both in vitro and in vivo.

\subsection{Is Super-Symmetry $\cong$ Field of Consciousness?}

It has already been explained that the existence of matter depends on the existence of force and vice versa. TCP cannot exist without TRP and vice versa. Many physicists believe that unifying all the forces, including gravity, into a single theory would require a phenomenon called supersymmetry. With super-symmetry, every fermion would have a boson twin, and vice-versa. The thought force $\left(\mathrm{T}_{\mathrm{F}}\right)$ is carried by the TCP in the presence of its super-symmetrical partner TRP. TCP that behaves like boson should accompany its super-symmetrical partner TRP that functions like fermion in the generalized simpler way. It is to be noted that these TCP and TRP function like wavicle: wave-particle duality.

Pal [14] has shown that 'thought' is a kind of force being carried by the TCP. A TCP has the capacity to carry a specific 'thought'. This TCP is converted into a TRP in the presence of consciousness to retain and store a specific 'thought' (or 'idea') as both these TCP and TRP are interchangeable in the presence of consciousness.

Thus, in the presence of consciousness, these TCP and TRP are inter-convertible to carry and retain a specific "thought" and also for its communication from one person to another in the presence of the thought force $\left(\mathrm{T}_{\mathrm{F}}\right)$ in vitro and thought force $\left(\mathrm{T}_{\mathrm{F}}\right)$ in vivo. It is to be taken into account that thought force $\left(\mathrm{T}_{\mathrm{F}}\right)$ is an expression of the universal consciousness.

Pal [15] has shown that both the TCP and TRP are interchangeable in the presence of consciousness, of course, in the inherent presence of the Soul ( $\cong$ Void which is the source of infinite energy) to complete the process of observation in order to see the observable 'object'.

Pal [16] expressed: It is to be noted that these TCP and TRP are interchangeable at super-symmetry and they function like wavicle: wave-particle duality. Pal [5] expressed that both the TCP and TRP have the same quantized energy and they behave like biophotons in animals and like simple photons in inanimate objects.

Now the Plausible Question is: Is SuperSymmetry $\cong$ Field of Consciousness?

Pal et al $[1,3]$ showed that the universe exists along with the universal consciousness. The quantized energy $\left(\varepsilon_{T}\right)$ of TCP is responsible to cause the universal consciousness. The individual consciousness owes its origin to the universal consciousness created by the same $\varepsilon_{T}$.

As both the TCP and TRP are interchangeable in the presence of consciousness, so it appears that the field of consciousness should function like super-symmetry; otherwise the inter-conversion between the TCP and TRP would not be possible.

\section{Plausible Possible Answers to the Typical Specific Questions Raised by Different Learned Reviewers}

\subsection{Question Number 1: How does the Thought Force cause an Acceleration, by $F=m A$ ?}

\section{Possible Answer:}

5.1.1. Pal et al [3] and Pal [5] explained that the quantized energy $\left(\varepsilon_{T}\right)$ of TCP is the energy responsible for generating thought force $\left(\mathrm{T}_{\mathrm{F}}\right)$; thus the $\mathrm{T}_{\mathrm{F}}$ may be expressed as

$$
T_{F}=\varepsilon_{T} / D_{i}
$$

where $D_{i}=$ Interacting distance.

The thought force $\left(\mathrm{T}_{\mathrm{F}}\right)$ is carried by TCP in the inherent 
presence of its super-symmetrical partner TRP. The TCP being the carrier of $\mathrm{T}_{\mathrm{F}}$ would behave like bosons when TRP would function like fermions.

Depending on the $D_{i}$ (= Interacting distance), we can calculate and identify two new forces viz. $\mathrm{T}_{\mathrm{F}}$ (micro) [= Thought force in microcosm] and $\mathrm{T}_{\mathrm{F}}$ (macro) [= Thought force in macrocosm], the existence of which is indicated and expressed by Pal [5].

When $\varepsilon_{T}=4.384 \times 10^{-16}$ erg and $D_{i}=r_{T n}=8.28 \times 10^{-18} \mathrm{~cm}$ in microcosm, it is found from the Equation (12) that

$$
\mathrm{TF}(\text { within a nucleon in microcosm })=\varepsilon_{T} / D_{i}=52.94 \text { dyne }\left(\text { within } 10^{-18} \mathrm{~cm}\right) .
$$

It is found theoretically that the radius of a TCP or TRP within a nucleon $\left(r_{T n}\right)=8.28 \times 10^{-18} \mathrm{~cm}$.

Thus $\mathrm{T}_{\mathrm{F}}$ (micro) $\equiv 52.94$ dyne $\cong 10^{5}$ SNF where $\mathrm{T}_{\mathrm{F}}$ (micro) $=$ Thought force in microcosm.

\section{The details of $T_{F}$ (micro) are as follows:}

$$
\begin{aligned}
& T_{F}(\text { micro }) \cong 10^{5} S N F \cong 10^{44} \mathrm{GF} \cong 52.94 \text { dyne } \\
& 33.04 \times 10^{12} \mathrm{eV} \cong 33.04 \times 10^{3} \mathrm{GeV} \cong
\end{aligned}
$$

Strongest interaction (at $D_{i}=10^{-18} \mathrm{~cm}$, the theoretically calculated radius of a TCP or TRP within a nucleon) where $\mathrm{T}_{\mathrm{F}}($ micro $)=$ Thought force in microcosm.

Again, when $\varepsilon_{T}=4.384 \times 10^{-16} \mathrm{erg}$ and $D_{i}=R$ (radius of the present universe) $=1.482 \times 10^{29} \mathrm{~cm}$ in the macrocosm, it is found from the Equation (12) that $\mathrm{T}_{\mathrm{F}}$ (in macrocosm) $=$ $\varepsilon_{T} / D_{i}=2.958 \times 10^{-45}$ dyne.

\section{The details of $T_{F}$ (macro) are as follows:}

$$
\begin{aligned}
& T_{F}(\text { macro }) \cong 10^{-3} \mathrm{GF} \cong 10^{-42} \mathrm{SNF} \\
& \cong 2.958 \times 10^{-45} \mathrm{dyne} \cong 1.846 \times 10^{-33} \mathrm{eV} \\
& \cong 1.846 \times 10^{-42} \mathrm{GeV} \cong
\end{aligned}
$$

Long-range weakest interaction [at $D_{i}$ (Interacting distance) $=$ radius of the universe $\left.\approx 1.482 \times 10^{29} \mathrm{~cm}\right]$ where $\mathrm{T}_{\mathrm{F}}($ macro $)=$ Thought force in macrocosm.

It is apparent from the equation (12) that the magnitude of thought force $\left(\mathrm{T}_{\mathrm{F}}\right)$ varies depending on the interacting distance $(\mathrm{Di})$ if the value of the quantized energy $\left(\mathcal{E}_{T}\right)$ of TCP is more or less same as derived through different equations.

In classical mechanics, we know

$$
\mathbf{F}=m \mathbf{a} \rightarrow \mathbf{a}=\mathbf{F} / m
$$

where $\mathrm{F}$ is the net force acting on the body, $m$ is the mass of the body, and a is the center-of-mass acceleration.

In the case of $\mathrm{T}_{\mathrm{F}}$ (micro) $\equiv 52.94$ dyne (within $10^{-18} \mathrm{~cm}$ ), using $\mathrm{m}=\mathrm{m}_{\mathrm{T}}=4.871 \times 10^{-37} \mathrm{~g}$, we get a $($ acceleration $)=\mathrm{F} / \mathrm{m}_{\mathrm{T}}=52.94 / 4.871 \times 10^{-37}=10.8684 \times 10^{37}$ $\mathrm{cm} / \mathrm{sec}^{2}$.

Thus, in the case of $T_{F}$ (micro), a (acceleration) = $10.8684 \times 10^{37} \mathrm{~cm} / \mathrm{sec}^{2}$.

In the case of $\mathrm{T}_{\mathrm{F}}$ (macro) $=\varepsilon_{T} / D_{i}=2.958 \times 10^{-45}$ dyne [within $D_{i}=R$ (radius of the present universe)
$=1.482 \times 10^{29} \mathrm{~cm}$ in the macrocosm], using $\mathrm{m}=\mathrm{m}_{\mathrm{T}}=$ $4.871 \times 10^{-37} \mathrm{~g}$, we get a (acceleration) $=\mathrm{F} / \mathrm{m}_{\mathrm{T}}=$

$2.958 \times 10^{-45} / 4.871 \times 10^{-37}=0.6173 \times 10^{-8} \quad \mathrm{~cm} / \mathrm{sec}^{2}=$ $6.173 \times 10^{-9} \mathrm{~cm} / \mathrm{sec}^{2}$.

Thus, in the case of $T_{F}$ (macro), a (acceleration) = $6.173 \times 10^{-9} \mathrm{~cm} / \mathrm{sec}^{2}$.

In general case, the thought force $\left(\mathrm{T}_{\mathrm{F}}\right)=\varepsilon_{T} / D_{i}=$ $4.384 \times 10^{-16} \mathrm{erg} / \mathrm{Di}\left(=\right.$ in $\left.\mathrm{g} . \mathrm{cm} / \mathrm{sec}^{2}\right)=$ $4.871 \times 10^{-37} \mathrm{~g} . \mathrm{cm} / \mathrm{sec}^{2}$.

In general case, when $\mathrm{F}=4.871 \times 10^{-37} \mathrm{~g} . \mathrm{cm} / \mathrm{sec}^{2}$ and $\mathrm{m}=$ $\mathrm{m}_{\mathrm{T}}=4.871 \times 10^{-37} \mathrm{~g}$, we get a (acceleration) $=\mathrm{F} / \mathrm{m}_{\mathrm{T}}=$ $4.871 \times 10^{-37} \mathrm{~g} . \mathrm{cm} / \mathrm{sec}^{2} / 4.871 \times 10^{-37} \mathrm{~g}=1 \mathrm{~cm} / \mathrm{sec}^{2}$.

Thus, in general case, a (acceleration) $=1 \mathrm{~cm} / \mathrm{sec}^{2}$.

It is found that the acceleration changes vigorously when the value of force changes thoroughly keeping the mass (m) more less same.

\subsection{Question Number 2: How does a Thought-Carrying Particle Interact with, Say, an Electron?}

\section{Possible Answers:}

5.2.1. Pal [16] showed through calculation that the radius of a TCP in the presence of TRP within a nucleon $\left(\mathrm{r}_{\mathrm{Tn}}\right)=8.28 \times 10^{-18} \mathrm{~cm}$. It can be shown that the radius of a TCP in the presence of TRP within an electron $\left(\mathrm{r}_{\mathrm{Te}}\right)=$ $2.113 \times 10^{-16} \mathrm{~cm}$ (see Table-I: Theoretical characteristics of TCP in the presence of TRP in the Section-4).

We now know that quarks, leptons and other elementary particles are nothing but discrete packets of energy. Any elementary particle is nothing but the quantized "lumps of energy". Although objects appear as solid, their constituent parts are in a constant state of flux.

Depending on the conditional elementarity of particles, quarks and electrons at sufficiently high temperatures are thought to be constituted by "infraquarks" or "infraelectrons" indicated by Weisskopf [6]. It is possible that these 'infraquarks' or 'infraelectrons' are ultimately constituted by these TCP and TRP in the presence of $T_{F}$ (micro). In other words, all the quarks, leptons and other elementary particles are ultimately constituted by these TCP and TRP in the presence of $\mathrm{T}_{\mathrm{F}}$ (micro) as indicated by Pal [5].

Bremsstrahlung and inverse photo-electric effect indicated by Wehr [17] signify the existence of TCP and $\mathrm{TRP}$ in the presence of $\mathrm{T}_{\mathrm{F}}$ (micro).

Modern experiments have probed at length scales of about $10^{-17} \mathrm{~cm}$, but even at that scale there is nothing to indicate that the electron has any structure. Although, Maris 
[18] claims that electrons can also be split into fragments called 'electrinos' which are conceived here to be constituted by these TCP and TRP in the presence of $\mathrm{T}_{\mathrm{F}}$ (micro). Wehr [17] mentioned, "We have pointed out that radiation results when a charged body is accelerated. Therefore when an electron loses a large amount of energy by being decelerated, an energetic pulse of electromagnetic radiation is produced. This is an inverse photoelectric effect in which an electron produces a photon. This photon, in our view, is the liberated TCP and TRP with which an electron is assumed to be constituted in the presence of $\mathrm{T}_{\mathrm{F}}$ (micro). What is called "bremsstrahlung" indicated by Wehr [17] indicating the breaking of charged particles like electrons signifies "breaking radiation" in which an electron produces a photon. This photon is nothing but the liberated TCP and TRP with which an electron is assumed to be composed in the presence of $\mathrm{T}_{\mathrm{F}}$ (micro).

\subsection{Question Number 3: How do you Relate these TCP and TRP either to the Body of Literature on QM and Consciousness?}

\section{Possible Answers:}

\subsubsection{Pal [19] published an article, "Possible Bridging the Classical Physics and Quantum Physics through Consciousness"}

Pal [19] explained that consciousness $\left(=\varepsilon_{T}\right)$ plays significant role to form a possible gross bridge between the classical physics and quantum physics.

\subsubsection{Interpretation of the Possible Origin of Matter and Force}

Mermin [20] asked the following questions:

"Are fundamental theories still based on super-positions of states that evolve unitarily, or have the basic principle of quantum mechanics been replaced? If quantum mechanics has survived, have people reached a consensus on the solution to the interpretive problems, or have they simply ceased to view them as problems needing a solution? If quantum mechanics has not survived, has the theory that replaced it clarified these puzzles, or do people find it equally or even more mysterious? Has any progress been made in understanding the nature of conscious experience or how the mind affects the body, and does quantum mechanics or its successor play a fundamental role in that understanding? Did quarks turn out to be elementary or composite? If composite, did the candidates for their constituents turn out to be elementary or composite? Or do we have a better way of looking at these phenomena?"

Physicists determined that underlying quantum fields give birth to elementary particles. Pal [5] explained that thought force $\left(\mathrm{T}_{\mathrm{F}}\right)$ being the primordial quantum field gives birth to TRP that appears to be the origin of all the matter particles. TCP is the carrier of thought force $\left(T_{F}\right)$ that, in turn, appears to be the origin of all the fields. TCP thus appears to be the origin of all the field particles.

\subsubsection{Phenomenology or Role of Consciousness?}

Phenomenology (from Greek: phainómenon "that which appears"; and lógos "study") is the study of the structure of experience. Phenomenology, in Husserl's conception, is primarily concerned with the systematic reflection on and study of the structures of consciousness and the phenomena that appear in acts of consciousness. Phenomenology (in particle physics) is a branch of particle physics that deals with the application of theory to high-energy experiments.

Why the micro world follows quantum rules and the macro one classical? A quantum description of aggregates of billions of atoms would provide a seamless elegant picture of nature. What prevents such uniformity? Where does the transition take place, and is it sudden?

It has been expressed that TCP is the origin of all the field particles and TRP is the origin of all the matter particles. One TRP (i.e. matter particle $\cong$ fermion) combines with another TRP in the presence of $\mathrm{T}_{\mathrm{F}}$ (micro) that, in turn, is carried by TCP (i.e. field particle $\cong$ boson). These TCP and TRP are the ultimate constituents to constitute a particle in the presence of $\mathrm{T}_{\mathrm{F}}$ (micro) as indicated by $\mathrm{Pal}$ [5]. It is possible that there is a critical limit of existence of these associated TRP in the presence of $\mathrm{T}_{\mathrm{F}}$ (micro). Up to certain extent, these associated TRP in the presence of $T_{F}$ (micro) will obey the quantum rules after which these associated TRP in the presence of $\mathrm{T}_{\mathrm{F}}$ (micro) would have to follow classical rule. Consciousness that, in turn, is the quantized energy $\left(\varepsilon_{T}\right)$ of TCP exerts its inherent functions throughout the universe and this function of consciousness is to be taken into account but usually ignored.

When a particle being constituted by these postulated TCP and TRP in the presence of $\mathrm{T}_{\mathrm{F}}$ (micro) combines with other particles to form a macroscopic object, the aggregate of particles would be liable to interact with the environment that upsets the delicate balance of quantum states; and the associated combination of these TCP and TRP at a certain level may be responsible to cause this disturbance through the functioning of consciousness. This consciousness, in turn, is the $\mathcal{E}_{T}$ (= quantized energy of the TCP) and thus makes a cat alive or dead but not in between through the utilization of consciousness. Consciousness thus plays significant roles both in the micro world (that follows quantum rules) and in the macro world (that follows classical rules). It is our plausible opinion that consciousness $\left(=\varepsilon_{T}\right)$ plays significant role to form a possible gross bridge between the classical physics and quantum physics.

Schrödinger (1935) described a thought experiment in which a cat is placed in a box into which poison is released when triggered by a particular quantum event. Schrödinger pointed out that according to the Copenhagen interpretation, the cat would be both dead and alive until the box was opened and the cat observed by a conscious human. Schrödinger cat is the first instance (Thought Experiment) to show a relation between mind (consciousness) and matter in terms of probability (uncertainty). But the present physics has not yet been able to solve the riddle of consciousness. 


\subsection{Question Number 4: What happens to my thought particles when I type or speak? Is someone producing or throwing particles? Are there dimensions for these particles?}

\section{Possible answers:}

\subsubsection{It will be Better if we first Try to Understand "Mode of Perception"}

Regarding the perception, let us see Scheme-III for the explanation expressed by Sânkhya psychology as indicated by Vivekananda [21]. In the case of vision, for instance, there are, first of all, the instruments of vision, the eyes. Behind the instruments (eyes), there is the organ of vision or 'Indria' (Sense organ), the optic nerve and its centers which are not the external instruments but without which the eyes will not see. The external sensations cannot be carried to the brain by the eyes alone if the sensory nerves (here optic nerve) and its centers are destroyed. Still more is needed for the perception. The mind (or Manas in Sanskrit) must come and attach itself to the organ to play its own role. Let us suppose that we are absorbed in reading a detective novel with close attention and if a bell rings at that time, we will not perhaps, hear the bell ring. The pulsation of that sound came to our ears, struck the tympanum; the impression was carried by the nerve into the brain. If the whole process was completed up ---catching, carrying and conducting the impulse to the brain, then why did we not hear? Something else was missing in the connection---the mind was not attached to the organ. When the mind detaches itself from the organ, the organ may bring any news to it, but the mind will not receive it. When mind attaches itself to the organ, then alone it is possible for the mind to receive the news. Yet, even that does not complete the whole circuit. The instrument may bring the sensation from the outside, the organ may carry it inside, the mind may attach itself to the organ, and yet the perception may not be complete. One more factor is necessary. There must be a reaction within. With this reaction comes knowledge. The sensation must be carried to the intellect (or BUDDHI), the determinative, reactive state of mind. The mind, after receiving the current of news from the brain, presents it to the intellect, which groups it in relation to the pre-received impressions and sends a current of reaction and perception becomes possible with that reaction. When the reaction comes from the intellect, along with it flashes the external world and egoism. Here then is the will. But everything is not complete. One more step is required. All the ideas in the mind must be gathered and projected on something that is stationary relatively to the body and mind. This internal 'something' stationary upon which our sensations, carried by the mind and intellect, are placed and grouped and formed into a unity, is

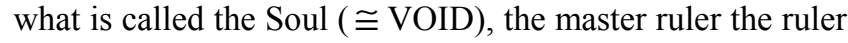
of the body (see Scheme-III). The impulse is presented to

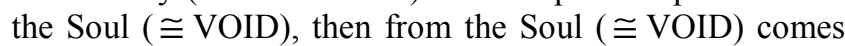
the order which goes down in the same sequence to the intellect, to the mind, to the brain, and the brain conveys it to the instruments through efferent nerve pathways and then the perception is truly complete (see Scheme-III).

According to the Sânkhya psychology as indicated by Vivekananda [21], the reactive state of mind called intellect (or BUDDHI) is the out-come or a certain manifestation of the cosmic mind (MAHAT) called the Universal Mind (UM).

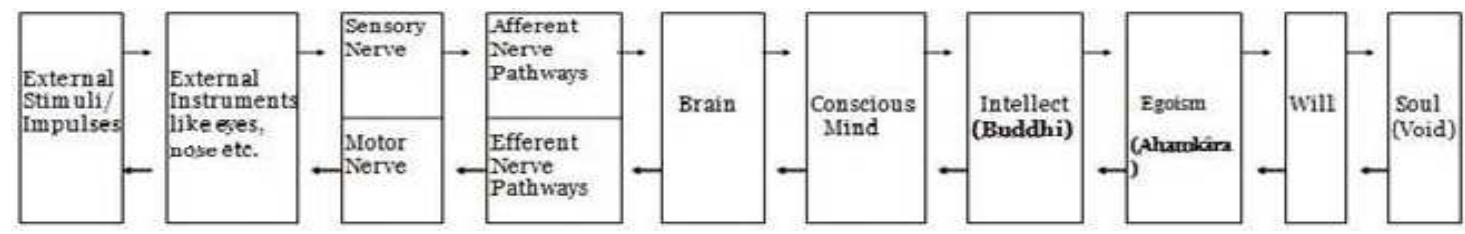

Scheme III. Schematic presentation showing the mode of perception: $(\rightarrow)$ indicates Sensory/Afferent Nerve conduction towards the brain. $(\leftarrow)$ indicates Motor/Efferent Nerve conduction towards periphery.

Pal [14] explained that the quantum mechanical activities of TCP and TRP in the inherent presence of the thought force $\left(\mathrm{T}_{\mathrm{F}}\right)$ in vitro and thought force $\left(\mathrm{T}_{\mathrm{F}}\right)$ in vivo play significant roles not only in information processing but also in any processing of task.

Pal et al $[1,3]$ explained that the universe exists along with the universal consciousness. This universal consciousness is a functional state of the Universal Mind (UM). This UM is constituted by these TCP and TRP which, in turn, are the ultimate constituents of any matter as well as any mind in the inherent presence of thought force $\left(\mathrm{T}_{\mathrm{F}}\right)$ in vitro and thought force $\left(\mathrm{T}_{\mathrm{F}}\right)$ in vivo. It is to be noted that these TCP and TRP are the ultimate constituents of any matter in the inherent presence of thought force $\left(\mathrm{T}_{\mathrm{F}}\right)$ in vitro. And these TCP and TRP are the ultimate constituents of any mind in the inherent presence of thought force $\left(\mathrm{T}_{\mathrm{F}}\right)$ in vivo.
When I type or speak or perform any job, I use my mind and my brain with other parts of my nervous system, of course, in the presence of consciousness. It will be wise to see the possible answers to Question Number 5, 6 and 7 in the next part.

Are there dimensions for these particles (i.e. TCP and TRP)? Yes. Theoretical characteristics of these TCP in the presence of TRP can be found in Table-I: Theoretical characteristics of TCP in the presence of TRP (see the Section- 4).

\subsection{Question Number 5: What are the Characteristic Functions of Soul ( $\cong$ VOID), Consciousness, Mind, Brain and Matter?}

\section{Possible Answers:}




\subsubsection{Characteristic Functions of Soul ( $\cong$ VOID), Consciousness, Mind, Brain and Matter}

Consciousness model of Pal et al [1-3] involving TCP, TRP and thought force $\left(T_{F}\right)$ signifies the existence of universal consciousness that exists along with the universe. Pal et al [3] showed that this universal consciousness is a functional state of the Universal Mind (UM). Pal et al [3] explained that the UM is evolved at the Big Bang from the eternal Void. The UM is constituted by these TCP and TRP in the inherent presence of thought force $\left(T_{F}\right)$. This thought force $\left(\mathrm{T}_{\mathrm{F}}\right)$ is an expression of universal consciousness.

These TCP and TRP, the constituents of the UM and the ultimate constituents of matter and mind are conceived here to be originated from the eternal Void at the Big Bang to evolve the space-time continuum and the UM along with the universal consciousness.

Pal et al [3] explained that the eternal Void has been covered by the mind to function as the Soul of the individual being. In the universe, behind the UM, there is a Soul ( $\cong$ VOID). In the individual, behind the individual mind (which is a constituent of the UM), there is also a Soul ( $\cong$ VOID).

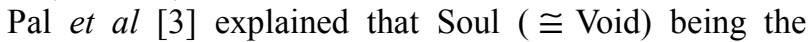
master ruler of the body exerts its functions through the finer instrument called mind. The mind (with the help of TCP and TRP) exerts its functions through the brain and spinal cord (CNS) along with the other parts of the nervous system (PNS along with ANS) in the biological systems in order to grasp this physical universe, of course, in the inherent presence of consciousness. This consciousness itself is acting as an inter-linking agent between the animate and inanimate through the quantum mechanical activities of these TCP and TRP. Consciousness is defined here as the 'self-organized' capability of any living being to activate TCP and TRP, the ultimate constituents of mind and matter. This consciousness, in turn, is the quantized energy $\left(\varepsilon_{T}\right)$ of TCP.

Any matter as well as the individual mind is constituted by these TCP and TRP that, in turn, are originated from the same eternal Void. Thus the individual mind is operated by these TCP and TRP that, in turn, are ultimately governed by the same eternal Void. The individual mind being a constituent of the UM has covered the eternal Void to serve the role of individual Soul. This eternal Void is thus the common source and the source is still with us indicated by Bhaumik [4]. This Void serves the role of Soul being covered by the mind. The mind-brain and brain-body links which are addressed by psychology, cognitive science, neuroscience and neurophysiology are, in our proposition, ultimately governed by the quantum mechanical activities of these TCP and TRP in the inherent presence of thought force $\left(\mathrm{T}_{\mathrm{F}}\right)$ in vitro and thought force $\left(\mathrm{T}_{\mathrm{F}}\right)$ in vivo. Consciousness that signifies the realization of existence is a functional state of mind.

\subsection{Question Number 6: Is there any Relationship between Mind and Consciousness, between Mind and Matter and between Consciousness and Matter? Possible Answers:}

\subsubsection{Interrelationship among Matter, Mind and Consciousness}

One of the central notions of human existence has been that of a Being prior to space, time, and substance. The world described by science must be a picture of mindindependent reality not as it is seen through the selective and deforming lens of our own sensory and mental structures through the inexact filter of consciousness. It seems we are forced to acknowledge that we cannot know mind-independent reality as it is.

Is there any relationship between the matter and consciousness? Can matter become conscious or can consciousness manifest itself in matter? The answer is always affirmative as the human being himself is the prominent example as indicated by Pal et al [1]. Does this signify that consciousness is generated from the matter? The affirmative answer in this case is a type of purely mind-made concept or belief. There is no scientific proof that consciousness is originated from the matter. The modern cognitive neuroscientists and Artificial Intelligence (AI) scientists think that 'minds are simply what brains do' and there always exists an inherent neural connection between the action of mind and nervous system. According to this concept of neuroscience, every physical reality is the result of a purely 'mind-made' concept having connections to nervous system. We must properly know and detect the exact 'vehicle' or 'agent' through which we are able to know. Whether consciousness is originated from the matter or not is possible to be asked and understood only in the presence of conscious mind alone. How is it possible to conclude that 'consciousness is originated from the matter' without gaining the proper knowledge of the exact characteristics of consciousness itself? Matter appears as matter only in the presence of consciousness but we do not yet know exactly what 'consciousness' itself really is. It cannot also be scientifically proved that consciousness does not originate from the matter. If 'mind' is accepted to be a type of fine matter, then and then only both the concepts about the manifestation of consciousness can be dealt with under 'materialism', otherwise everything concerning consciousness as well as mind is liable to come under 'idealism'.

Logically, the substantial view of mind is to be accepted to proceed further in the consciousness study. In this view, the mind is a type of substance. Mind is a finer matter having an autonomous existence. Mind is a single entity, perhaps having its base in the brain but distinct from it.

Note: What is the exact definition of mind? What is mind?

We observe a great controversy concerning the exact definition of mind. There are two apparently opposite views: one is the substantial view and the other is the functional view.

In the substantial view, the mind is a type of substance. Mind is a finer matter having an autonomous existence. Mind is a single entity, perhaps having its base in the brain but distinct from it. In its most extreme form as in the Indian Upanishads and Vedanta, the mind is not only a finer matter but also it is an entity wholly separate from the body, in fact a manifestation of the soul, which will survive the 
body's death in the form of the spiritual body or mental body [called Linga Sharira or Sukshma Sharira in Sanskrit] as expressed by Vivekananda [21] who indicated that this mental body bears all the mental impressions. This mental body is also called 'spirit'.

In the functional view, the mind is closely related to the functions of the brain and can have no autonomous existence beyond the brain, nor can they survive its death. In this view, mind is a 'state' created by the activities of the brain with other parts of the nervous system as it is indicated by cognitive neuro-scientists and Artificial Intelligence (AI) scientists. According to them 'minds are simply what brains do'. In this functional view, mind is a subjective manifestation of consciousness: the human brain's ability to be aware of its own existence. The concept of the mind is therefore a means by which the conscious brain understands its own operations.

Modern Scientists are not yet able to understand how the brain works to make the mind. They know that brain has got neurons that communicate across synapses by releasing a neurotransmitter, and that generates electrical impulses, and the receiving neuron then talks to its neighbor neurons the same way. If the mind depends on the brain, then all aspects of the mind are going to depend on these simple electrical, chemical processes. According to this concept, the existence of the UM requires the existence of the universal brain to form the UM. The concept of universal brain is not logically acceptable. Thus the concept that the mind depends on the brain becomes questionable if the existence of the UM is valid.

Wigner [22] became interested in the Vedanta philosophy of Hinduism, particularly its ideas of the universe as an all pervading consciousness. He commented "It was not possible to formulate the laws (of quantum theory) in a fully consistent way without reference to consciousness."

Wigner [22] also conceived the Wigner's friend thought experiment in physics, which is an extension of the Schrödinger's cat thought experiment. The Wigner's friend experiment asks the question: "At what stage does a 'measurement' take place?" Wigner designed the experiment to highlight how he believed that consciousness is necessary to the quantum-mechanical measurement processes.

\subsection{Question Number 7: Is there any Relation between Quantum Mechanics and Brain in Order to Understand the Interrelationship among Consciousness, Mind, Brain and Matter?}

\section{Possible Answers:}

\subsubsection{Relation between Quantum Mechanics and Brain: Interrelationship among Consciousness, Mind, Brain and Matter}

Discussions of the roles of quantum mechanics that might or might not play in the theory of consciousness/mind have become increasingly sharp. One side of this debate stands for the conventional neuroscientists who assert that brain science must look to the neuron for understanding, and on the other side are certain physicists, suggesting that the rules of quantum theory might influence the dynamics of consciousness/mind However, consciousness and mind are not separate from matter. The interrelationship among consciousness, mind, brain and matter are to be established thoroughly. Mind as well as consciousness owes its origin to Submicroscopic aspect of the human brain. We are unable to make a sharp separation between mind and matter. Thus ultimately there is no "mind" that can be separated from "matter" and no "matter" that can be separated from "mind". The brain is an interface between mind and matter and brain functions as a mixed physical system composed of the macroscopic neuron system and an additional microscopic system. The former consists of pathway conduction of neural impulses. The latter is assumed to be a quantum mechanical manybody system interacting with the macroscopic neuron system.

All these aspects can be explained through the existence of the quantum mechanical activities of these TCP and TRP, the ultimate constituents of any matter as well as any mind, in the inherent presence of thought force $\left(\mathrm{T}_{\mathrm{F}}\right)$ in vitro and the thought force $\left(\mathrm{T}_{\mathrm{F}}\right)$ in vivo indicated by $\mathrm{Pal}$ et al $[1,3]$.

The reigning tenet of quantum mechanics is the uncertainty principle. A consequence of the uncertainty principle is that the presence of an observer or experimenter determines the outcome of the observation or experiment. Simply stated, this means there is no objective reality; you 'create' what you see through the quantum mechanical activities of these TCP and TRP in the presence of consciousness. The quantized energy $\left(\varepsilon_{T}\right)$ of TCP represents universal consciousness. The individual consciousness owes its origin to the universal consciousness created by the same $\varepsilon_{T}$. Pal et al $[1,3]$ explained that these TCP and TRP are the ultimate constituents of any matter as well as any mind in the inherent presence of thought force $\left(\mathrm{T}_{\mathrm{F}}\right)$ in vitro and the thought force $\left(\mathrm{T}_{\mathrm{F}}\right)$ in vivo.

How could an object's existence depend upon the act of observation? This is due to the fact that an object's existence as well as the act of observation through the prevailing consciousness is totally dependent on the existence of the quantum mechanical activities of these TCP and TRP in the presence of consciousness. The quantized energy $\left(\varepsilon_{T}\right)$ of TCP represents universal consciousness. The individual consciousness owes its origin to the universal consciousness created by the same $\varepsilon_{T}$. These TCP and TRP are the ultimate constituents of any matter as well as any mind in the inherent presence of thought force $\left(\mathrm{T}_{\mathrm{F}}\right)$ in vitro and the thought force $\left(\mathrm{T}_{\mathrm{F}}\right)$ in vivo as indicated by Pal et al $[1,3]$.

\section{Discussion and Conclusion}

\subsection{Discussion}

The existence of TCP, TRP and thought force $\left(\mathrm{T}_{\mathrm{F}}\right)$ in vitro and thought force $\left(\mathrm{T}_{\mathrm{F}}\right)$ in vivo may provide plausible guidelines to lucidly explain the evolution of the universe 
with fundamental laws of natural science like physics, chemistry and biology (with consciousness).

Pal et al [1-3] explained that the thought force $\left(\mathrm{T}_{\mathrm{F}}\right)$ and the developed consciousness model may provide plausible guidelines to form a relationship of consciousness with other well-defined matter. This consciousness model may also provide guidelines to tackle many present day scientific enigmas.

It appears that the standard model of physics is to be correctly tuned by ascertaining the constituents of quarks and leptons in the presence of unified field in order to form a possible gross bridge between mind and matter, present physics and cognitive science, psychology and natural sciences, classical physics and quantum physics.

\subsection{Conclusion}

Theoretical characteristics of TCP and TRP can provide possible answers to many typical questions. Here possible answers to seven typical questions are addressed.

\section{References}

[1] D. Pal and A.U. De, Physics of consciousness and its model may provide guidelines to solve Many scientific problems. Neuroquantology 1: 17-28(2004)

[2] D. Pal and A.U. De, Consciousness model: Significance of thought-carrying particles and thought-retaining particles in quantum measurement as well as cognitive problem. Neuroquantology 2: 115-116 (2005)

[3] D. Pal and A.U. De, The cosmic microwave background radiation temperature signifying the existence of the thought-carrying particle, thought retaining particle and thought force. NeuroQuantology 10: Issue3; 428-442 (September 2012)

[4] M. Bhaumik, Code Name GOD. (Penguin Books India Pvt. Ltd., 11 Community Centre, Panchsheel Park, New Delhi 110017 , India) pp. 132-133; 161-162; 167; 171; 177; 183 and 184. 185-186; 89-190; 198 (2006)

[5] Dhananjay Pal, Existence of Thought Force and Its Characteristics, American Journal of Modern Physics. Vol. 2, No. 6, 2013, pp. 364-374. doi: 10.11648/j.ajmp.20130206.23

[6] V. F. Weisskopf, The Origin of the Universe, The World of Physics, (Simon and Schuster, 1230 Avenue of Americas, New York 10020) 3, pp. 10 and 314 (1987)

[7] D. Pal, Existence of universal consciousness and its characteristics. Accepted for publication in 2014 International Conference on Advanced Education and Management (ICAEM2014) Beijing, China (2013)

[8] I. Prigogine and G. Nicolis, Self-Organization in NonEquilibrium Systems. (Wiley 1977) ISBN 0471024015.
[9] R. Nelson, Director, Global Consciousness Project, Princeton, New Jersey. (2009) rdnelson@princeton.edu

[10] D. Radin, The Conscious Universe: The Scientific Truth of Psychic Phenomena. (Harper Edge) (1997) ISBN 0-06251502-0.

[11] R. Penrose, Shadows of the Mind: A Search for the Missing Science of Consciousness. (Oxford University Press) (1994)

[12] W. Van De Bogart, Earth portals: Exploring New Metaphors of Consciousness 1993 willard@earthportals.com

[13] G. Gamow, The Great Expansion, The World of Physics, (Simon and Schuster, 1230 Avenue of Americas, New York 10020, New York 10020) 3:271 (1987)

[14] Dhananjay Pal, Interpretation of Information Processing and Mode of Perception Through the Consciousness Model Involving Though-Carrying Particle (TCP), Thought Retaining Particle (TRP) and Thought Force (TF) in vitro and Thought Force (TF) in vivo, American Journal of Modern Physics. Vol. 2, No. 6, 2013, pp. 341-349. doi: 10.11648/j.ajmp.20130206.21

[15] Dhananjay Pal, Interpretation of Quantum Measurement as Well as Cognitive Problem through the Consciousness Model, American Journal of Modern Physics. Vol. 2, No. 6, 2013, pp. 375-381. doi: 10.11648/j.ajmp.20130206.24

[16] Dhananjay Pal, Application of the Consciousness Model Involving Thought-Carrying Particle (TCP), Thought Retaining Particle (TRP) and Thought Force (TF) to Explain the Cause of Radioactivity, American Journal of Modern Physics. Vol. 2, No. 6, 2013, pp. 392-395. doi: 10.11648/j.ajmp.20130206.27

[17] MR. Wehr, Richards JA. Jr and Adair T W. Physics of the Atom, Narosa Publishing House, 6, Community Centre, Panchseel Park, New Dilhi 110017; Fourth Edn.; 224- 225 and 519 (1996)

[18] HJ.Maris, on the fission of elementary particles and the evidence for fractional electrons in liquid helium. Journal of Low Temperature Physics 2000; 120: 173-204.

[19] Dhananjay Pal, Possible Bridging the Classical Physics and Quantum Physics through Consciousness, American Journal of Modern Physics. Vol. 2, No. 6, 2013, pp. 322-329. doi: 10.11648/j.ajmp.20130206.18

[20] N. David Mermin, What's wrong with These Questions? Physics Today 11 (February 2001) http://www.physicstoday.org

[21] Swami Vivekananda, the Vedanta Philosophy. The real and the apparent Man. The complete works of Swami Vivekananda. (Advaita Ashrama 1989) 1: P-359-360; The Real and the Apparent Man, ibid.; 2: P-263.

[22] E. Wigner. Symmetries and Reflections: Scientific Essays. MIT Press. (1970) 